\section{Н.И.Марьенко А.Ю.Степаненко}

Харьковский национальный медицинский университет

Ключевые слова: человек, мозжечок, индивидуальная анатомическая изменчивость.

Надійшла: 23.01.2017

Прийнята: 10.03.2017
DOI: https://doi.org/10.26641/1997-9665.2017.1.45-50

УДК: 611.817 .1

\section{СТРОЕНИЕ И ИНДИВИДУАЛЬНАЯ АНАТОМИЧЕСКАЯ ИЗМЕНЧИВОСТЬ ДВУБРЮШНОЙ ДОЛЬКИ МОЗЖЕЧКА ЧЕЛОВЕКА}

Исследование проведено в рамках научно-исследовательской работы "Строение и закономерности индивидуальной анатомической изменчивости головного мозга человека" (номер государственной регистрации 0115U000231).

Реферат. Цель исследования - изучить закономерности индивидуальной изменчивости и вариантную анатомию дольки VIII полушарий мозжечка человека. Исследована форма двубрюшной дольки полушарий мозжечка человека, описаны 3 варианта формы: 1-й вариант: белое вещество дольки имеет форму буквы Y; 2-й вариант: белое вещество в виде буквы V; 3-й вариант: белое вещество дольки включает две отдельные ветви. Наиболее распространенным вариантом формы ростральной части является 3-й вариант, а каудальной части - 1-й.

Morphologia. - 2017. - T. 11, № 1. - C. 45-50.

(С) Н.И.Марьенко, А.Ю.Степаненко, 2017

$\triangle$ kafedra-histology-knmu@mail.ua

\begin{abstract}
Maryenko N.I., Stepanenko O.Yu. Structure and individual anatomical variability of the biventral lobule of the human cerebellum.

ABSTRACT. Background. Morphological changes of the cerebellar lobules are found in many congenital and acquired diseases of the cerebellum, but the information on the normal structure of the cerebellar lobules do not take into account the peculiarities of individual anatomical variability, sex and age characteristics. Objective - to investigate features of individual variability and variant anatomy of the lobule VIII of the human cerebellar hemispheres. Methods. The study was conducted on 100 cerebellums of people of both sexes, who died of causes unrelated to brain pathology (20-95 years old). Features of the shape, branching of white matter of lobule VIII, number, shape and location of folia of gray matter on parasagittal sections of the cerebellar vermis were investigated. Results. Complex branched white matter is the basis of the VIII lobule of human cerebellar hemispheres. Lobule is divided into two parts: lobule VIIIA (rostral part) and VIIIB (caudal part). The main branch of the white matter is dichotomously divided into different number of secondary branches. We described 3 variants of branching of white matter of lobule VIII. First variant of lobule consists of main trunk of white matter, which is divided into two secondary branches. The white matter is Y-shaped. Second variant of lobule includes two branches of white matter which are connected only in the basal part. The white matter is V-shaped. Third variant of lobule includes two branches of white matter which are not connected. The most common variant forms of the rostral part lobule VIII is the 3rd variant, and the caudal part - 1st variant. Conclusion. Described variants of the shape of the cerebellar lobule VIII can be used as criteria standards of modern diagnostic imaging techniques for the diagnosis of various diseases of the CNS.
\end{abstract}

Key words: human, cerebellum, individual anatomical variability.

Citation:

Maryenko NI, Stepanenko OYu. [Structure and individual anatomical variability of the biventral lobule of the human cerebellum]. Morphologia. 2017;11(1):45-50. Russian.

\section{Введение}

В последние годы благодаря современным методам нейровизуализации (МРТ, фМРТ, КТ, ОФЭКТ, ПЭТ) были обнаружены морфологические изменения долек мозжечка (изменение объема долек, объема и структуры серого и белого вещества) при различных психических заболеваниях - аутизме, синдроме дефицита внимания с гиперактивностью, дислексии, шизофрении, биполярных расстройствах [1-13]. Патологические изменения VIII-й дольки червя и полушарий мозжечка найдены у больных с аутизмом и ши- зофренией $[8,10,11,13]$. Морфологические изменения долек червя и полушарий мозжечка встречаются также при многих других врожденных и приобретенных заболеваний мозжечка (наследственной мозжечковой атаксии Пьера Мари, цереброоливарной атрофии Холмса, мозжечковой атрофии Мари-Фуа-Алажуанина, оливопонтоцеребеллярний дегенерации, синдромах Денди-Уокера, Арнольда-Киари IV, болезни Альцгеймера, рассеянном склерозе, алкогольной мозжечковой дегенерации) [5, 14-16]. Патологические изменения долек полушарий и червя 
мозжечка, которые встречаются при этих заболеваниях, могут быть обнаружены прижизненно, что необходимо для ранней и точной диагностики [14, 17]. Однако сведения о нормальном строении долек мозжечка, на которых базируются критерии нормы диагностических методов нейровизуализации [18-20], не учитывают особенностей индивидуальной анатомической изменчивости, половых и возрастных особенностей. В связи с этим актуальным направлением современной нейроморфологии является исследование нормального строения и особенностей индивидуальной анатомической изменчивости долек полушарий и червя мозжечка человека. Ранее нами были исследованы строение и закономерности индивидуальной анатомической изменчивости VIII дольки червя мозжечка человека [21], однако строение соответствующих долек полушарий отличается от долек червя и требует отдельного рассмотрения.

Цель исследования - изучить закономерности индивидуальной изменчивости и вариантную анатомию дольки VIII полушарий мозжечка человека.

\section{Материал и методы}

Исследование проведено на базе Харьковского областного бюро судебно-медицинской экспертизы на 100 объектах - мозжечках трупов людей обоего пола, умерших от причин, не свя- занных с патологией мозга, в возрасте 20-95 лет.

В ходе судебно-медицинского вскрытия проводили морфометрию мозжечка после его выделения из черепной коробки, затем его фиксировали в течение месяца в $10 \%$-м растворе формалина. После этого проводили рассечение червя мозжечка строго в центральной сагиттальной плоскости. Затем производили серийные парасагиттальные срезы полушарий в плоскостях, параллельных срединной сагиттальной плоскости с пошаговым интервалом 5 мм. Вид мозжечка на срезах фотографировали с помощью зеркального цифрового фотоаппарата, после чего проводили анализ оцифрованных изображений.

Изучались особенности формы дольки VIII полушарий мозжечка, структура её белого и серого вещества, определялись места окончания ветвей дольки. В связи с тем, что производились серийные парасагиттальные срезы на расстоянии 5 мм друг от друга, как место окончания ветви принималось расстояние от срединной сагиттальной плоскости до наиболее латерального среза, на котором эта ветвь присутствует $(5,10$, 15, 20, 25, 30, 35, 40 мм).

\section{Результаты и их обсуждение}

Двубрюшная долька полушарий мозжечка (VIII долька, lobulus biventer) разделяется на две части: дольку VIIIA (ростральная часть) и VIIIB (каудальная часть) (рис. 1).

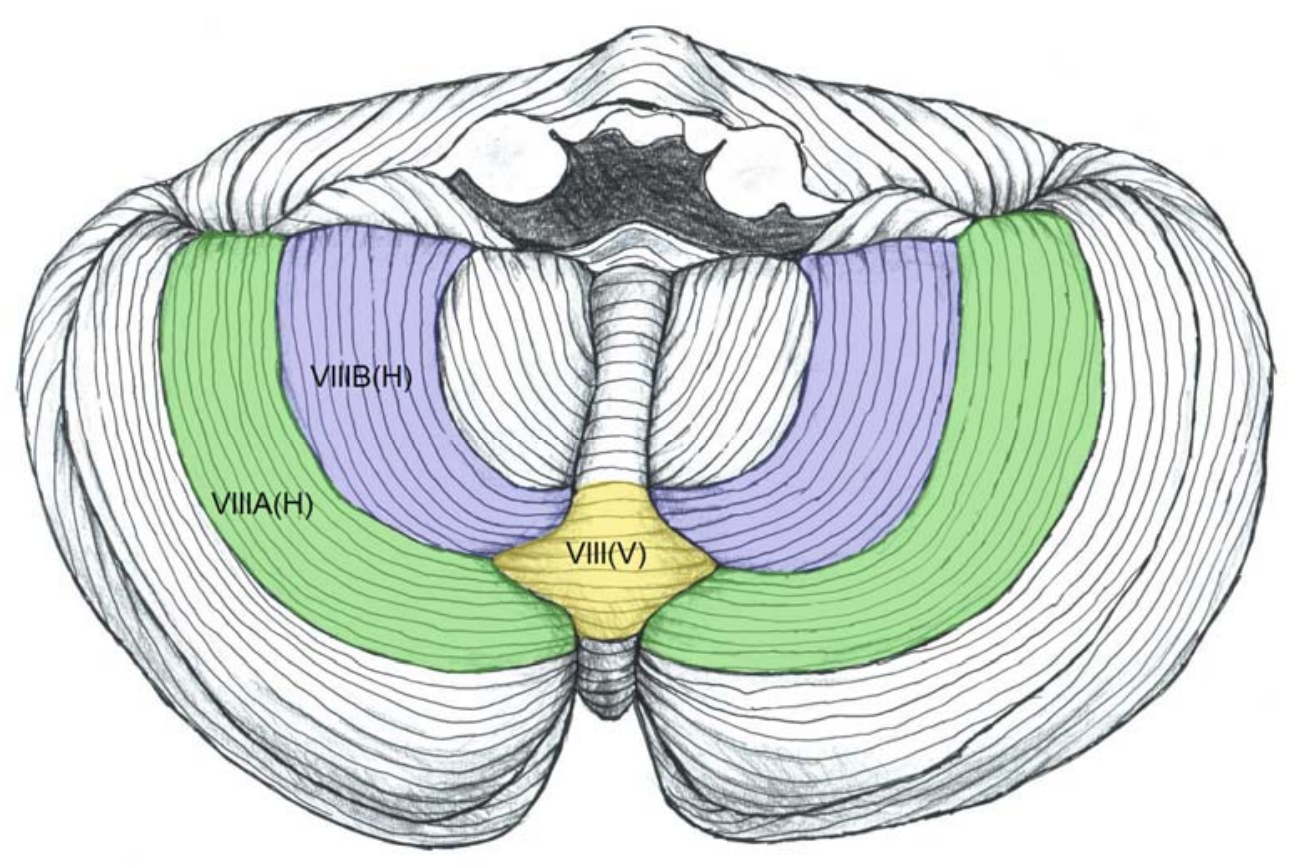

Рис. 1. Нижняя поверхность мозжечка. VIIIA(H) - рострально-латеральная часть двубрюшной дольки (заштрихована зеленым цветом); VIIIB(H) - каудально-медиальная часть (заштрихована синим цветом); VIII(V) - пирамида червя (заштрихована желтым цветом).

От тонкой дольки двубрюшную дольку отделяет препирамидная щель (Fissura prepyramidalis), от миндалины - вторичная щель (fissura secunda). Две части двубрюшной дольки (VIIIA и
VIIIB) между собой разделены fissura intrabiventralis. Обе части двубрюшной дольки начинаются в паравермальных (медиальных) участках полушарий, продолжаются в латераль- 
ном направлении, затем отклоняются кпереди, огибая миндалины. Таким образом, в медиальных участках извилины двубрюшной дольки расположены перпендикулярно срединной сагиттальной плоскости, а в латеральных участках - параллельно. Медиально в паравермальных участках полушарий двубрюшная долька соединяется с долькой VIII червя (Руramis, Пирамида) с помощью тонкой пластинки, которая лежит глубоко между извилинами и иногда четко не различима. В отличие от I-VII долек, извилины и белое вещество дольки червя не переходит непосредственно в дольки полушарий.

На серийных парасагиттальных срезах долька имеет вид трапеции (рис. 2). У дольки есть три поверхности: свободная (является частью види- мой поверхности мозжечка), ростральная (верхняя) и каудальная (нижняя).

Обе части двубрюшной дольки имеют похожую форму и одинаковый план строения. Белое вещество состоит двух крупных ветвей, которые могут отходить от мозгового тела мозжечка по отдельности или общим стволом. Место разделения главного ствола на основные ветви может быть на разном расстоянии от центральной сагиттальной плоскости. Основные ветви белого вещества дольки дихотомически разделяются на разное количество дочерних ветвей.

Учитывая особенности структры белого вещества VIIIA и VIIIB долек полушарий мы выделили три варианта строения долек (рис. 2).

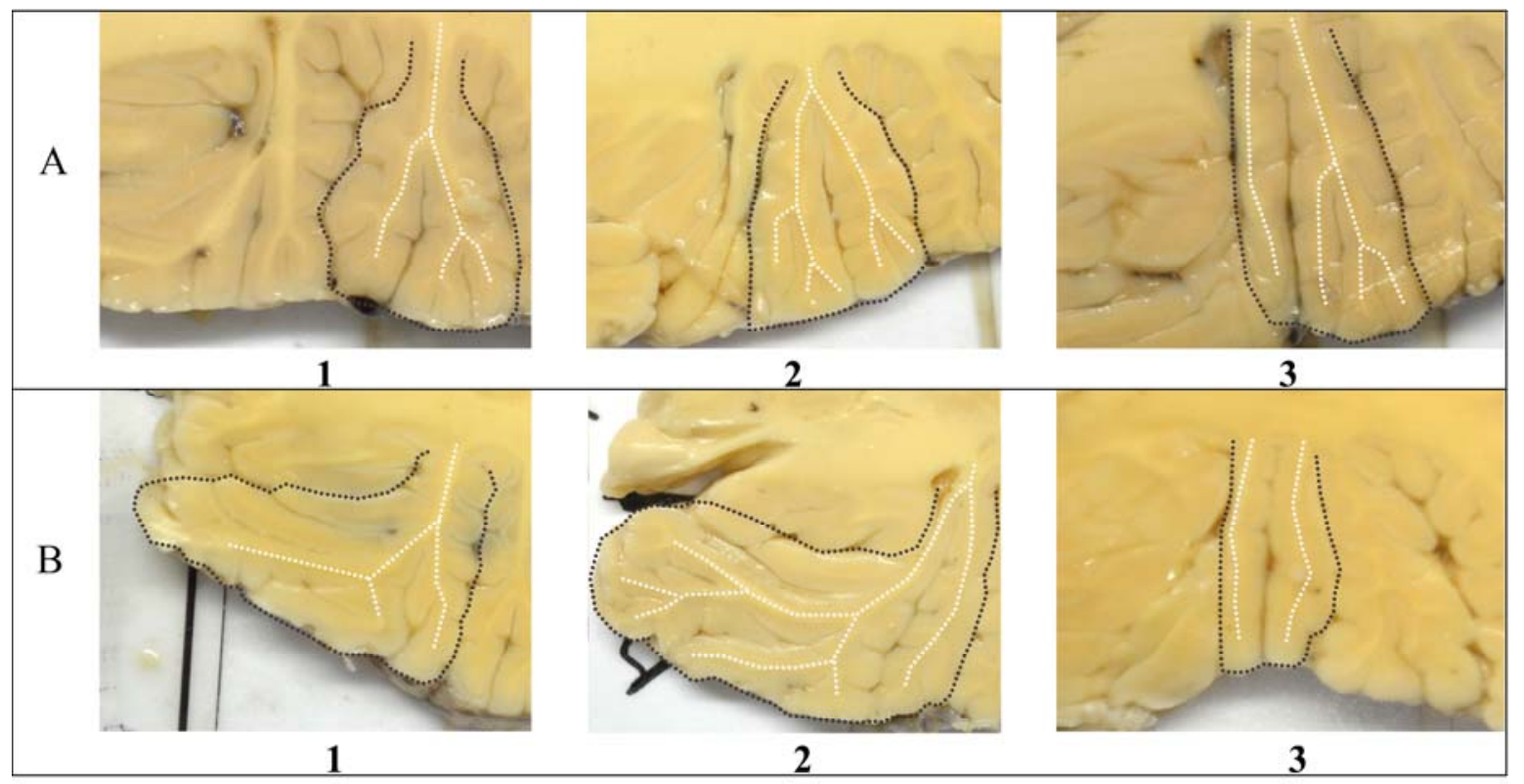

Рис. 2. Варианты строения дольки VIII полушарий мозжечка человека $(1,2,3)$, правое полушарие. Черным пунктиром обозначены границы долек, белым пунктиром - ход основных ветвей белого вещества дольки.

A - долька VIIIA, 10 мм от срединной сагиттальной плоскости;

B - долька VIIIB, 15 мм от срединной сагиттальной плоскости.

Первый вариант. Главный ствол белого вещества ближе к видимой поверхности мозжечка разделяется на две дочерние ветви; белое вещество дольки имеет форму буквы Y.

Второй вариант. Две основные ветви белого вещества соединяются в базальной части в виде буквы V.

Третий вариант. Белое вещество представлено двумя отдельными ветвями белого вещест- ва, самостоятельно отходящими от мозгового тела мозжечка.

Как видно из данных таблицы 1, наиболее распространенным вариантом формы ростральной части дольки VIII является третий вариант, а каудальной - первый. При этом в ростральной части варианты справа и слева совпадают в $61 \%$ наблюдений, а в каудальной части - в 77\% наблюдений.

Таблица 1

Частота встречаемости вариантов строения дольки VIII полушарий мозжечка человека (\%)

\begin{tabular}{lcccc}
\hline \multirow{2}{*}{ Вариант формы } & \multicolumn{2}{c}{ Долька VIIIA } & \multicolumn{2}{c}{ Долька VIIIB } \\
\cline { 2 - 5 } & Левое полушарие & Правое полушарие & Левое полушарие & Правое полушарие \\
\hline 1 & 12 & 9 & 54 & 53 \\
2 & 40 & 43 & 37 & 39 \\
3 & 48 & 48 & 9 & 8 \\
\hline
\end{tabular}


Таблица 2

Сочетание вариантов разветвления белого вещества ростральной и каудальной частей дольки VIII полушарий мозжечка человека

\begin{tabular}{lcccc}
\hline \multirow{2}{*}{ Долька VIIIB } & \multicolumn{3}{c}{ Долька VIIIA } \\
\cline { 2 - 5 } & 1 & 2 & 3 & Сумма \\
\hline 1 & 11 & 7 & 3 & 21 \\
2 & 44 & 32 & 7 & 83 \\
3 & 52 & 37 & 7 & 96 \\
Сумма & 107 & 76 & 17 & 200 \\
\hline
\end{tabular}

Варианты разветвления белого вещества в ростральной и каудальной частях (VIIIA и VIIIB) совпадают в $25 \%$ (50 случаев).

В латеральных участках полушарий строение долек упрощается: уменьшаются количество и размеры листков серого вещества, уменьшает- ся толщина ветвей белого вещества. Ветви последовательно редуцируются и в латеральных участках исчезают - сначала мелкие, потом и главные. Распространенность мест окончания ветвей в латеральных участках полушарий приведена на рис. 3 .

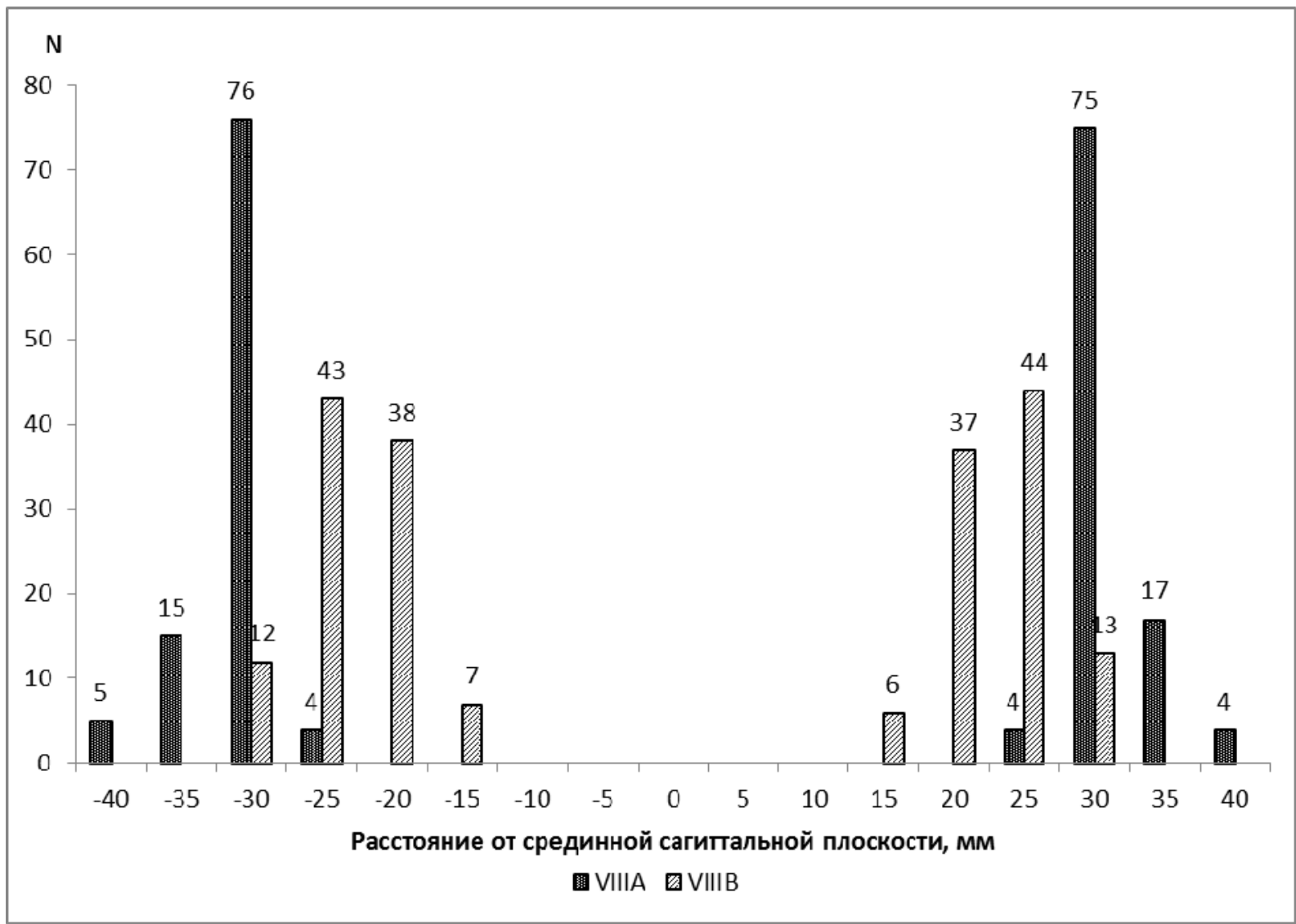

Рис. 3. Распространенность мест окончания долек VIIIA и VIIIB полушарий мозжечка человека (отрицательные значения оси абсцисс - левое полушарие, положительные значения - правое полушарие).

Как видно из данных рис. 3, ростральная часть двубрюшной дольки (VIIIA) заканчивается в латеральных участках полушарий (на расстоянии от 25 до 40 мм от срединной сагиттальной плоскости), а каудальная часть (VIIIB) - в медиальных участках (на расстоянии от 15 до $30 \mathrm{Mм}$ от срединной сагиттальной плоскости).

На особенности строения двубрюшной дольки влияют морфометрические параметры мозжечка. Наиболее выраженное влияние на место окончания дольки имеет ширина мозжечка, что отражается в разных значениях ширины мозжечков с разными метами исчезновения долек (рис. 4).

Как видно из данных рис. 4, в медиальных участках заканчиваются ветви двубрюшной дольки в мозжечках с меньшей шириной, латеральных участков полушарий эта долька дости- 
гает в мозжечках с относительно большими значениями ширины. Разница между средними значениями ширины у мозжечков с разными местами окончания ветвей двубрюшной дольки стати- стически значима, $\mathrm{P}<0,05$ (сравнение проводилось с помощью критериев Краскела-Уоллиса и Данна).

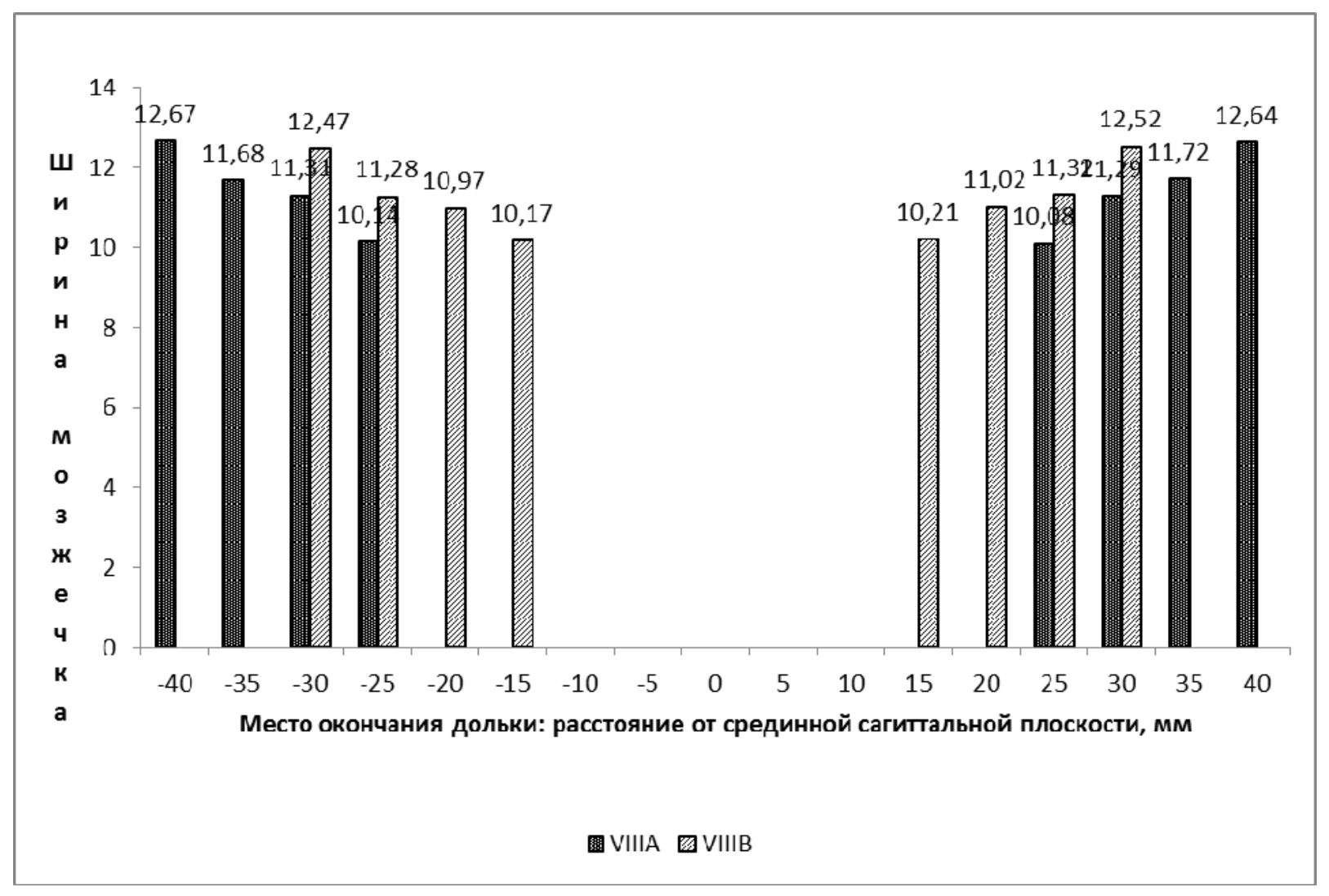

Рис. 4. Средняя ширина мозжечков (см) с разными местами окончания ветвей двубрюшной дольки (отрицательные значения оси абсцисс - левое полушарие, положительные значения - правое полушарие).

\section{Выводы}

Таким образом, ростральная и каудальная части VIII дольки полушарий мозжечка имеют одинаковый план и варианты строения. Описаны 3 варианта разветвления белого вещества ростральной и каудальной частей двубрюшной дольки; наиболее распространенным вариантом ростральной части является третий, а каудальной части - первый.

Особенности строения двубрюшной дольки связаны с шириной мозжечка: в более узких мозжечках ветви дольки заканчиваются в меди- альных участках полушарий, в широких мозжечках ветви достигают латеральных участков полушарий мозжечка.

\section{Перспективы дальнейших разработок}

Описанные варианты строения дольки могут быть использованы в качестве критериев нормы для диагностических методов нейровизуализации, а также для составления атласов серийных срезов мозжечка, составленных с учетом закономерностей индивидуальной анатомической изменчивости.

\section{Литературные источники References}

1. Anderson CM, Rabi K. Cerebellar lingula size and experiential risk factors associated with high levels of alcohol and drug use in young adults cerebellum. Neuroscience. 2010;9(2):198-209.

2. Berquin PC, Giedd JN, Jacobsen LK. Cerebellum in attention-deficit hyperactivity disorder A morphometric MRI study. Neurology.
1998;(50):1087-93.

3. Courchesne E, Saitoh O, Townsend JP, Yeung-Courchesne R, Press GA, Lincoln AJ. Cerebellar hypoplasia and hyperplasia in infantile autism. Lancet. 1994;343:63-4. doi:10.1016/S01406736(94)90923-7.

4. DelBello MP. MRI analysis of the cerebel- 
lum in bipolar disorder: a pilot study. Neuropsychopharmacology. 1999;21:63-8.

5. Dietrichs E. Clinical manifestation of focal cerebellar disease as related to the organization of neural pathways. Acta Neurol Scand Suppl. 2008;(188):6-11.

6. Fatemi SH, Aldinger KA, Ashwood P. Consensus paper: pathological role of the cerebellum in autism. Cerebellum. 2012;11(3):777-807.

7. Jeremy D, Schmahmann JD, Jeffrey B. The neuropsychiatry of the cerebellum - insights from the clinic. The Cerebellum. 2007;(6):254-67.

8. Levitt JG, Blanton R, Capetillo-Cunliffe L, et al. Cerebellar vermis lobules VIII-X in autism. Progress in Neuro-Psychopharmacology \& Biology Psychiatry. 1999;(23):625-33.

9. Montes LG, Ricardo-Garcell J, DelaTorre LB, et al. Cerebellar gray matter density in females with ADHD combined type: across-sectional voxelbased morphometry study. J Atten Disord. 2011;(15):368-81.

10. Russell T, Loeber BS, Christina MB, et al. Morphometry of individual cerebellar lobules in schizophrenia. Am J Psychiatry. 2001;(158):952-4.

11. Stoodley CJ. Distinct regions of the cerebellum show gray matter decreases in autism, ADHD, and developmental dyslexia. Frontiers in Systems Neuroscience. 2014;(92):2-17.

12. Varnäs K, Okugawa G, Hammarberg A, et al. Cerebellar volumes in men with schizophrenia and alcohol dependence. Psychiatry Clin Neurosci. 2007;61(3):326-9.

13. Yeganeh-Doost P, Gruber O, Falkai P,et al.
The role of the cerebellum in schizophrenia: from cognition to molecular pathways. Clinics (Sao Paulo). $2011 ; 66: 71-7$.

14. Basson MA, Wingate RJ. Congenital hypoplasia of the cerebellum: developmental causes and behavioral consequences Frontiers in Neuroanatomy. 2013;3:7-29.

15. Leonard JR, Ojemann JG. Dandy-Walker Syndrome. Youmans Neurological Surgery. 2004;3:3285-8.

16. Mauritz K, Dichgans J, Hufschmidt A. Quantitative analysis of stance in late cortical cerebellar atrophy of the anterior lobe and other forms of cerebellar ataxia. Brain. 1979;102:461-82.

17. Courchesne E, Press GA, Murakami J, et al. The cerebellum in sagittal plane - anatomic-MR correlation: the vermis. Am J Radiol. 1989;1(53):829-35.

18. Duvernoy HM, Naidich ThP, Delman BrN, et al. Duvernoy's atlas of the human brain stem and cerebellum. Wien, Springer-Verlag; 2009. 888 p.

19. Larsell O, Jansen J. The comparative anatomy and histology of the cerebellum. The human cerebellum, cerebellar connections, and the cerebellar cortex. Minneapolis: University of Minnesota Press; 1972. 268 p.

20. Voogd J, Glickstein M. The anatomy of the cerebellum. Trends Neurosci. 1998;21:370-5.

21. Stepanenko OYu, Maryenko NI. [Structural organization and variant anatomy of the lobule VIII of the human cerebellar vermis]. Ukrainian morphological Almanac. 2012;2(10):129-32. Russian.

Мар’єнко Н.І., Степаненко О.Ю. Будова та індивідуальна анатомічна мінливість двочеревцевої часточки мозочка людини.

Реферат. Мета дослідження - вивчити закономірності індивідуальної анатомічної мінливості і варіантні анатомію часточки VIII півкуль мозочка людини. Досліджена форма двочеревцевої часточки півкуль мозочка людини, описані 3 варіанти форми: 1-й варіант: біла речовина часточки має форму літери Y; 2-й варіант: біла речовина у вигляді букви V; 3-й варіант: біла речовина включає дві окремі гілки. Найбільш поширеним варіантом форми ростральної частини є 3-й варіант, а каудальної частини - 1-й.

Ключові слова: людина, мозочок, індивідуальна анатомічна мінливість. 